\title{
Modelling of damping force of dynamically loaded specimen of polyurethane foam in dependence on its density
}

\author{
David Cirkl* \\ Technical University of Liberec, Department of Mechanical Engineering, Studentska 2, Liberec 460 01, Czech \\ Republic
}

\begin{abstract}
Article deals with measurement and modelling of damping force of polyurethane foam specimen being compressed. The specimens were taken from car seats and differ by material density. For modelling of damping force during harmonical kinematical excitation the explicit formula is used. Its parameters are found by means of method based on evaluation of energy dissipated during one loading cycle and also on evaluation of position of damping force extreme.
\end{abstract}

Keywords: Passengers comfort, car seat, polyurethane foam, damping force

\section{Introduction}

The significant factors of comfort of a person sitting in a moving car are the statical and dynamical properties of seats whose cushioning is predominantly made from polyurethane (PU) foam. Therefore one of parameters by which it is possible to influence viscoelastic properties of seat is the density of PU cushioning material.

To research this influence three PU seat cushionings with different material density were made and from each seat the specimens with dimensions $(100 \times 100 \times 50) \mathrm{mm}$ were cut. By division of their mass by volume the material density was calculated for the three specimens marked A $\left(47 \mathrm{~kg} / \mathrm{m}^{3}\right), \mathrm{B}\left(56 \mathrm{~kg} / \mathrm{m}^{3}\right)$ and C $\left(63 \mathrm{~kg} / \mathrm{m}^{3}\right)$. Differences in material density are also evident from images made by electron microscope in Fig. 1.

\section{Loading of specimens}

The specimens were dynamically compressed by harmonical kinematical signal of displacement Eq. (1), where mean value $A_{0}=25 \mathrm{~mm}$, amplitude $A=5 \mathrm{~mm}$ with the frequency varying in values $f \in\{0.1,0.5,0.7,1,1.5,2,3,4$, $5\} \mathrm{Hz}$. Measurements were carried out under environment temperature (21-22) ${ }^{\circ} \mathrm{C}$ and relative humidity (50-60)\%.

$$
x(t)=A_{0}+A \sin (\omega t), \omega=2 \pi f .
$$

\footnotetext{
${ }^{*}$ Corresponding author. E-mail: david.cirkl@tul.cz.
} 


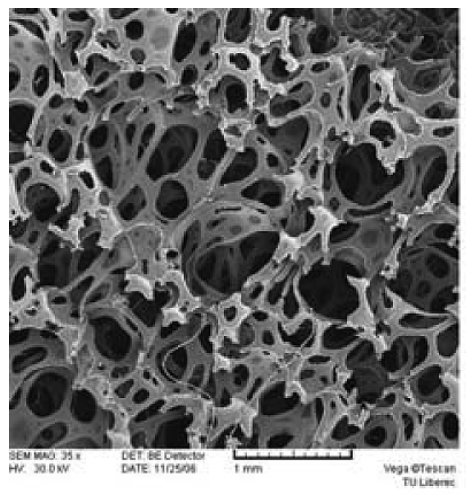

spec. A

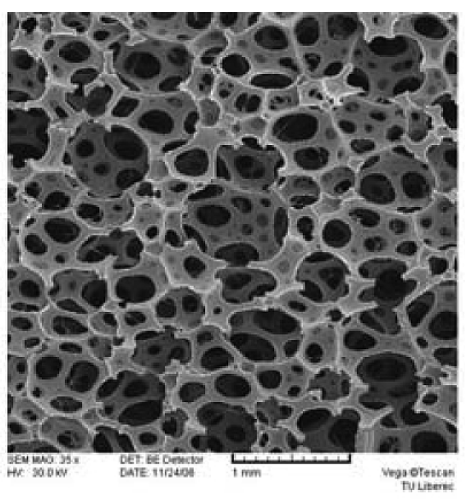

spec. B

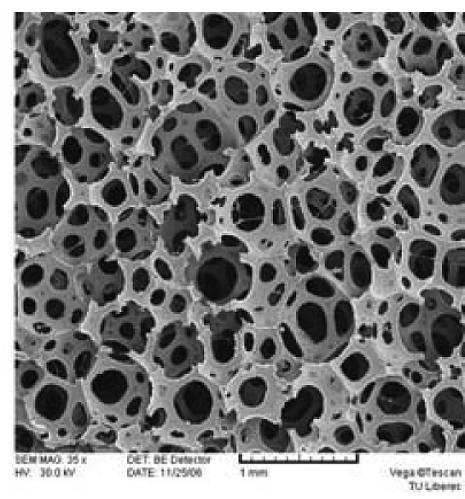

spec. C

Fig. 1. Structure of polyurethane foam specimens with different density.
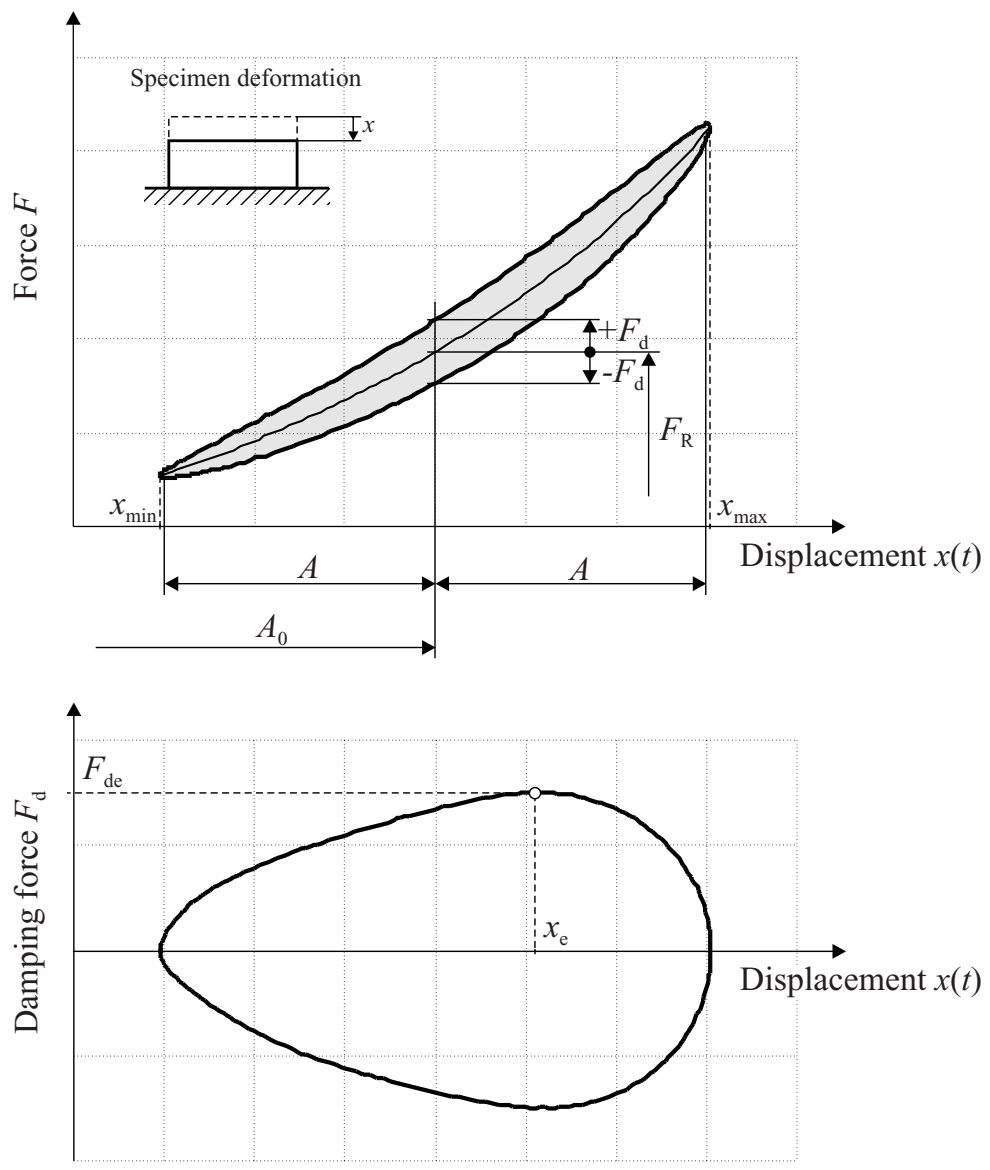

Fig. 2. Force decomposition.

\section{Methodology of measurement evaluation}

In case of dynamical compression of the PU foam specimen by harmonical displacement $x(t)$ for one period we get the hysteresis course of the measured total force $F$ as shown in Fig. 2 at the top. Let us assume that the damping force $F_{\mathrm{d}}$ is distributed around the skeleton curve of hysteresis loop symmetrically. Let us also assume that this 
Table 1

Average position of extreme of damping force, absolute and relative standard deviation

\begin{tabular}{ccccc}
\hline \multirow{2}{*}{ Quantity } & \multicolumn{3}{c}{ Specimens } & \multirow{2}{*}{ Compounded } \\
\cline { 2 - 4 } & $\mathrm{A}$ & $\mathrm{B}$ & $\mathrm{C}$ & \\
\hline $\bar{x}_{\mathrm{e}}[\mathrm{mm}]$ & 27.1231 & 27.0604 & 27.3555 & 27.1797 \\
$\Delta x_{\mathrm{e}}[\mathrm{mm}]$ & 0.96269 & 1.1098 & 0.65466 & 1.0061 \\
$\delta x_{\mathrm{e}}[\%]$ & 0.26111 & 0.30033 & 0.17909 & 0.27347 \\
\hline
\end{tabular}

skeleton curve represents just the restoring force $F_{\mathrm{R}}$. In general it has progressive nonlinear character and shows hardening behavior with increasing exciting frequency. In Fig. 2 at the bottom there is the course of damping force in dependence on displacement $x$. This dependency shows a typical pear-like character with significant extreme value $F_{\mathrm{de}}$ at position $x_{e}$.

Work of damping force (dissipated energy) is given as a curve integral of damping force $F_{\mathrm{d}}$ with respect to $x$ :

$$
W_{\mathrm{d}}=\oint F_{\mathrm{d}} \mathrm{d} x \text {. }
$$

\section{Measured quantities}

In Fig. 3 there are measured courses of total force response $F$ and damping force $F_{\mathrm{d}}$ in dependency on displacement $x$-e.g. for specimen B. Qualitatively same courses are also shown with specimens A and C. The statistical evaluation of measured position of extreme of damping force $x_{e}$ in relation to frequency change for individual specimens is presented in Table 1. With 50-fold change of frequency (from 0.1 to $5 \mathrm{~Hz}$ ) there is not a significant nor systematical change of this position. This statement is supported by the fact that average positions $\bar{x}_{\mathrm{e}}$ have relative standard deviations $\delta x_{\mathrm{e}}$ only from $0.65466 \%$ to $1.1098 \%$. Relative standard deviation in percent form is calculated as a standard deviation $\Delta x_{e}$ divided by mean value $\bar{x}_{\mathrm{e}}$ multiplied by 100 . So in accordance with previously published results in [1] or [2] we can say that position of extreme of damping force $\bar{x}_{e}$ is independent on exciting frequency. This is also valid even for the other two measured specimens. Moreover, average position $x_{e}$ calculated for all individual positions together for all three specimens A, B, C (in Table 1 denoted as compounded) gives approximately same values as calculations made for individual specimens. So for further application we can assume that $x_{e}$ is independent not only on exciting frequency but also even on material density, having constant value $\hat{x}_{\mathrm{e}}=27.1797$ mm (see Fig. 4).

Another important quantity is dependency of dissipation work of damping force $W_{\mathrm{d}}$ on exciting frequency and material density. Although the foam material is strongly nonlinear in space $(x, F)$, as mentioned by many authors (see [3,4]), it is not difficult to prove that the dependency of $W_{\mathrm{d}}$ on frequency can be considered as linear (see [1, 2]). So it is sufficient to consider polynomial of the first degree to be approximating function in this dependency. To express influence of material density there it is sufficient to consider polynomial of second degree for good approximation (see Fig. 5). This approximating function is denoted $\hat{W}_{\mathrm{d}}$ and was determined using least square method. At this point we can continue to damping force simulation.

\section{Simulation of damping force}

In technical handbooks as [5] or [6] it is possible to find that damping force of elastic elements, at which damping depends on displacement $x$ and is realized by energy dissipation inside the material, can be defined by Eq. (3). This formulation appears to be applicable in case of description of damping force of polyurethane foam being compressed.

$$
F_{\mathrm{d}}(x, \dot{x})=b_{\alpha}\left|x^{\alpha}\right| \dot{x} .
$$

Because the specimens were loaded by only positively oriented deformations (with respect to suitable coordinate system) it is possible to leave out the absolute value in Eq. (3) and rewrite it in form Eq. (4). The task then lies in finding the coefficient $b_{\alpha}$ and exponent $\alpha$.

$$
F_{\mathrm{d}}(x, \dot{x})=b_{\alpha} x^{\alpha} \dot{x} .
$$



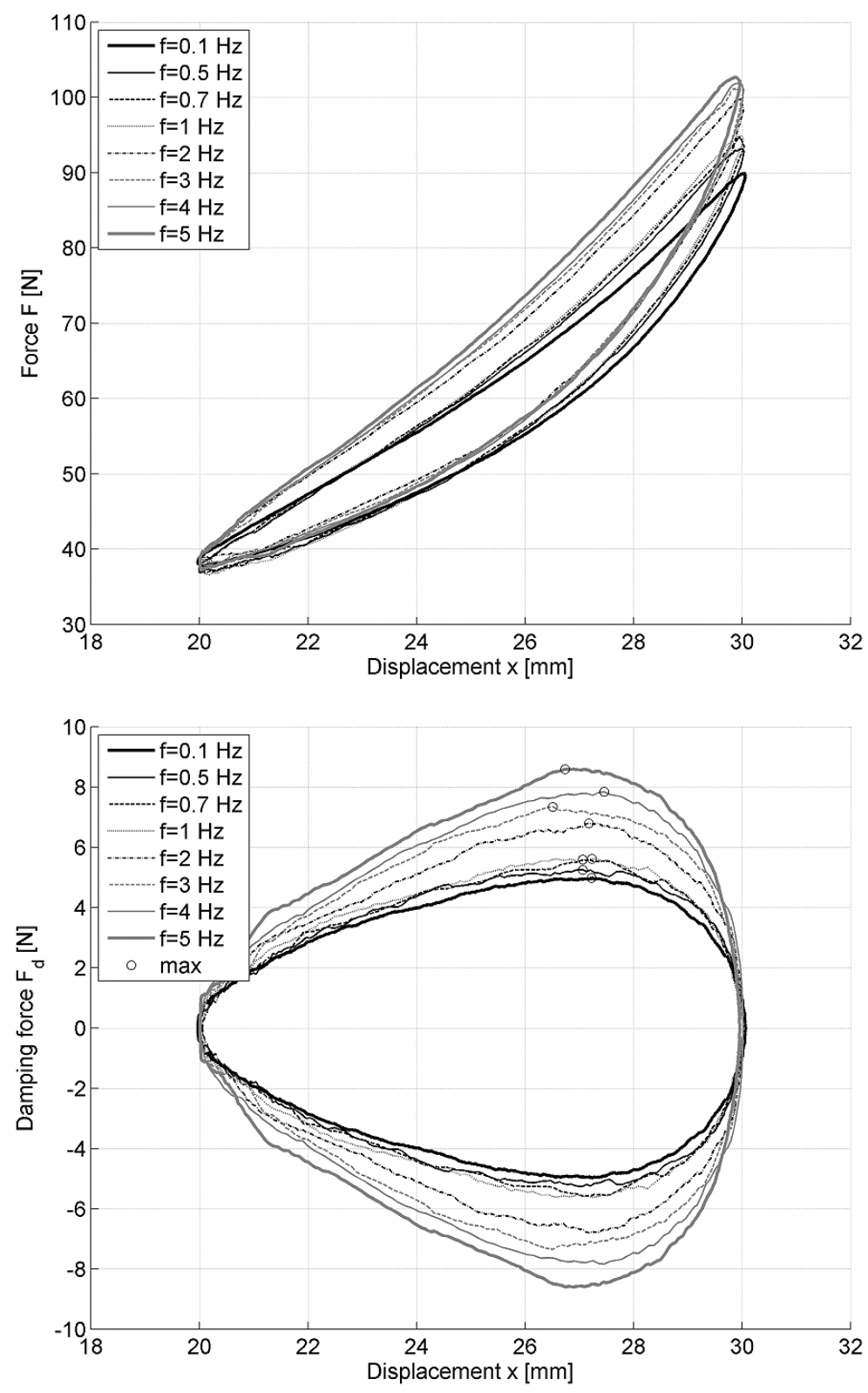

Fig. 3. Measured total and damping force (specimen B).

\subsection{Determination of $\alpha$ exponent}

To search for the exponent of function Eq. (4) we will find its extreme by putting its derivative with respect to $x$ equal to zero. If we have unequivocally assigned $x$ and $\dot{x}-\mathrm{e}$.g. by choosing certain time course - it is possible to assume the Eq. (4) as the function of one variable. Position of extreme is denoted as $x_{\mathrm{e}}$.

$$
\frac{\mathrm{d} F_{\mathrm{d}}}{\mathrm{d} x}=b_{\alpha}\left[\alpha x_{\mathrm{e}}^{(\alpha-1)}+x_{\mathrm{e}}^{\alpha} \frac{\mathrm{d} \dot{x}}{\mathrm{~d} x}\right]=0, \text { where } \frac{d \dot{x}}{d x}=\frac{\ddot{x}}{\dot{x}} .
$$

Thus we get the equation

$$
\frac{\alpha}{x_{\mathrm{e}}}+\frac{\ddot{x}}{\dot{x}^{2}}=0 .
$$




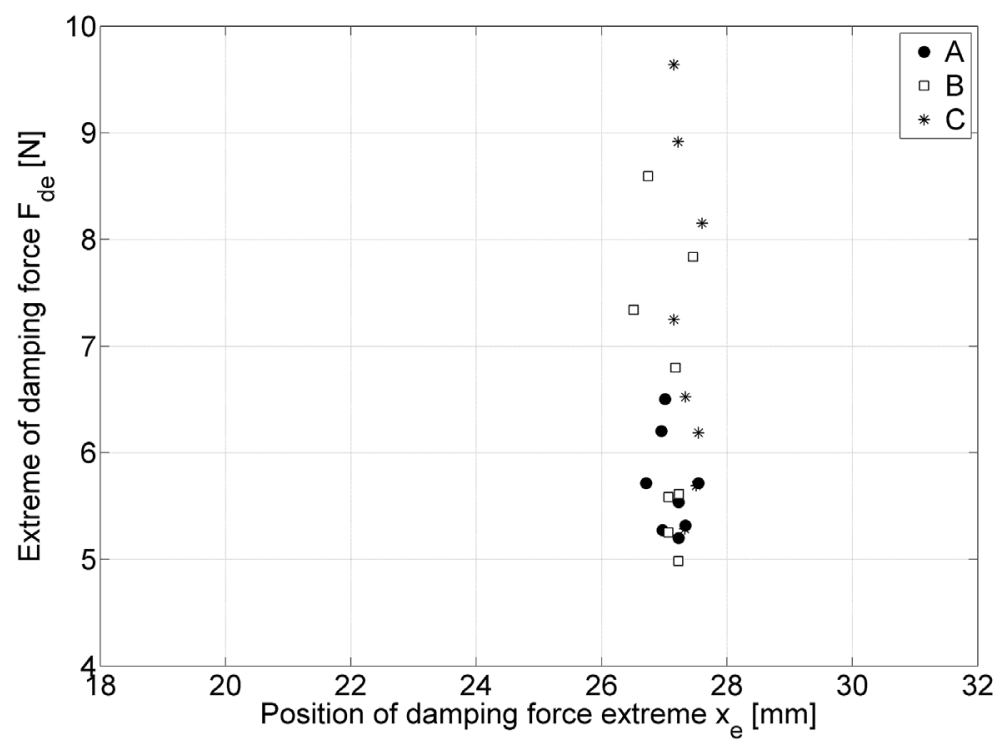

Fig. 4. Positions and values of damping force extreme.

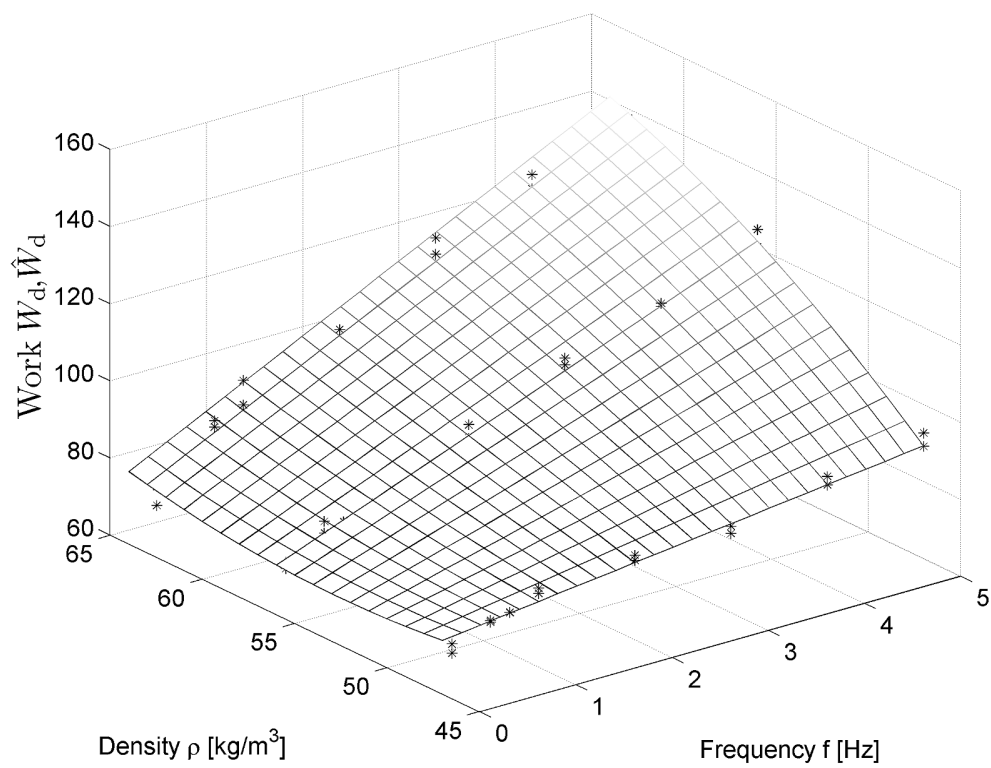

Fig. 5. Dependency of dissipation work of damping force $W_{\mathrm{d}}$ on frequency $f$ and material density $\rho\left(W_{\mathrm{d}}-\right.$ measured values $(*), \hat{W}_{\mathrm{d}}-$ approximation (surface))

Specially for harmonical function of displacement $x$ we get system of equations:

$$
\begin{aligned}
& x=A_{0}+A \sin (\omega t), \\
& \dot{x}=A \omega \cos (\omega t), \\
& \ddot{x}=-A \omega^{2} \sin (\omega t) .
\end{aligned}
$$

From this we can now express the velocity $\dot{x}$ and the acceleration $\ddot{x}$ as functions (9) and (10) depending only on displacement $x$. 


$$
\begin{aligned}
& \dot{x}(x)= \pm \omega \sqrt{A^{2}-\left(x-A_{0}\right)^{2}}, \\
& \ddot{x}(x)=-\omega^{2}\left(x-A_{0}\right) .
\end{aligned}
$$

We substitute these results into Eq. (5) where $x=x_{e}$ and we get

$$
\frac{\alpha}{x_{\mathrm{e}}}+\frac{\ddot{x}\left(x_{\mathrm{e}}\right)}{\dot{x}^{2}\left(x_{\mathrm{e}}\right)}=\frac{\alpha}{x_{\mathrm{e}}}+\frac{-\omega^{2}\left(x_{\mathrm{e}}-A_{0}\right)}{\omega^{2}\left[A^{2}-\left(x_{\mathrm{e}}-A_{0}\right)^{2}\right]}=0
$$

After canceling of square of angular velocity $\omega$ we obtain an implicit function (11) which it is possible to use for calculation of damping force extreme position. In this equation the angular velocity $\omega$, respectively exciting frequency $f$, is not present.

$$
\frac{\alpha}{x_{\mathrm{e}}}-\frac{\left(x_{\mathrm{e}}-A_{0}\right)}{A^{2}-\left(x_{\mathrm{e}}-A_{0}\right)^{2}}=0 .
$$

By this it has been proved that damping force formulation (4) does not show the dependency of extreme position on exciting frequency, which is in accordance with observations.

Because the position of extreme $x_{\mathrm{e}}$ is defined by approximating value $\hat{x}_{\mathrm{e}}$ it is possible to use the Eq. (11) for exponent $\alpha$ calculation in form Eq. (12). Another way of exponent $\alpha$ determination is described in [7].

$$
\alpha=\frac{\left(\hat{x}_{\mathrm{e}}-A_{0}\right)}{A^{2}-\left(\hat{x}_{\mathrm{e}}-A_{0}\right)^{2}} \hat{x}_{e} .
$$

\subsection{Determination of $b_{\alpha}$ coefficient}

As has already been written above the work of damping force $W_{\mathrm{d}}$ for one loading period is given by line integral of damping force $F_{\mathrm{d}}$ with respect to displacement $x$. In Eq. (2) we substitute Eq. (4) for $F_{\mathrm{d}}$ and we get:

$$
W_{\mathrm{d}}=\oint F_{\mathrm{d}} \mathrm{d} x=b_{\alpha} \oint x^{\alpha} \dot{x} \mathrm{~d} x=b_{\alpha} \int_{0}^{2 \pi / \omega} x^{\alpha}(t) \dot{x}^{2}(t) \mathrm{d} t .
$$

Using time courses Eqs (6) and (7) where differential $\mathrm{d} x=A \omega \cos (\omega t) \mathrm{d} t$, and substituting $\omega t=\varphi$ we transform this integration into angular displacement domain $\varphi$. Under consideration of constant angular velocity $\omega$ during one loading period we can write:

$$
W_{\mathrm{d}}=b_{\alpha} \omega \int_{0}^{2 \pi}\left[A_{0}+A \sin (\varphi)\right]^{\alpha}[A \cos (\varphi)]^{2} \mathrm{~d} \varphi .
$$

Using expression $\omega=2 \pi f$ we rewrite this equation into form

$$
W_{\mathrm{d}}=b_{\alpha} 2 \pi f I_{\alpha}
$$

where

$$
I_{\alpha}=I_{\alpha}\left(A_{0}, A, \alpha\right)=\int_{0}^{2 \pi}\left[A_{0}+A \sin (\varphi)\right]^{\alpha}[A \cos (\varphi)]^{2} \mathrm{~d} \varphi
$$

is an integral independent on frequency of harmonical exciting signal. As Eq. (14) shows the work of damping force $W_{\mathrm{d}}$ depends on frequency linearly which is in accordance with experimental observation. Value of integral Eq. (15) is for given mean value, amplitude and $\alpha$ exponent computed numerically. From Eq. (14) we express the coefficient $b_{\alpha}$ where we substitute work $W_{\mathrm{d}}$ for our purpose by approximation $\hat{W}_{\mathrm{d}}$ :

$$
b_{\alpha}=\frac{\hat{W}_{\mathrm{d}}}{2 \pi f I_{\alpha}} \text {. }
$$




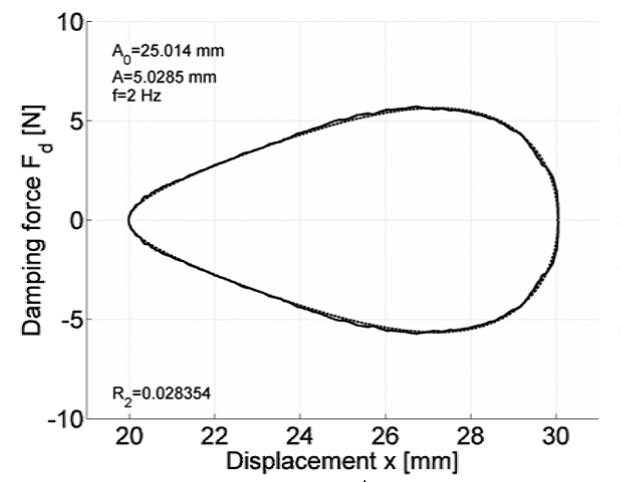

spec. A

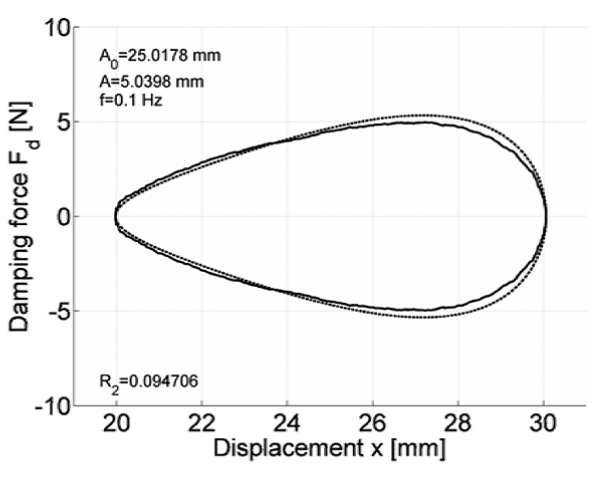

spec. B

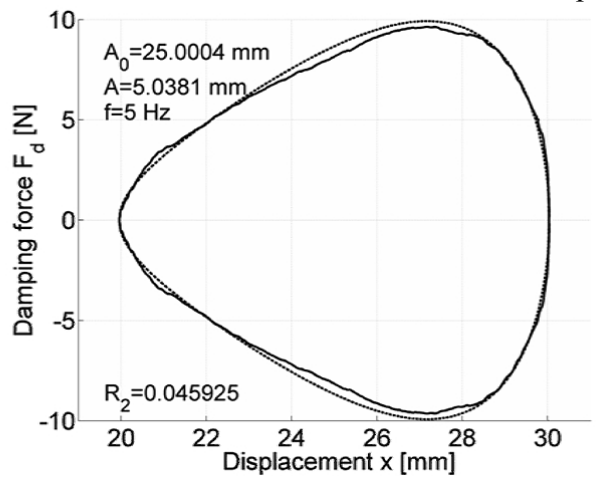

spec. C

Fig. 6. Results of simulation (Measurement - solid line, Simulation - dotted line).

\section{Achieved results}

\subsection{Comparison of measured and simulated damping force}

Equation (4) has been calculated for all combinations of exciting frequency and material density. Selected instances are pictured in Fig. 6. Simulated damping force was calculated using real (measured) displacement $x$. Because of this reason in each graph there is noted real mean value and amplitude for which the data were acquired and which are different from desired theoretical values. This difference is given by control deviation of experimental device. Velocity of displacement $\dot{x}$ then has been calculated under consideration of harmonical signal.

For quantification of simulation error there has been defined quantity $R_{2}$ given by Eq. (17), where $S_{\mathrm{e}}$ is summation of deviations squared and $S$ is summation of measured values squared. $F_{\mathrm{dm} i}$ here is the denoted measured value of damping force and $F_{\mathrm{ds} i}$ is corresponding sample of simulated value. The quantity $R_{2}$ has been evaluated in interval $x \in\left\langle x_{\min } ; x_{\max }\right\rangle ; n$ is number of measured samples in this interval. Values of $R_{2}$ for all cases lies in interval [0.0284, 0.0947] and are plotted in Fig. 7 in detail.

$$
R_{2}=\sqrt{\frac{S_{\mathrm{e}}}{S}}, \quad S_{e}=\sum_{i=1}^{n}\left(F_{\mathrm{dm} i}-F_{\mathrm{ds} i}\right)^{2}, \quad S=\sum_{i=1}^{n} F_{\mathrm{dm} i}^{2}
$$

\subsection{Damping force prediction in dependence on material density}

Up to this point the method of damping force simulation has been used only in cases really measured. But we can use it in entire domain of definition of quantities $W_{d}$ and $x_{e}$, which means for $f \in[0.1 ; 5] \mathrm{Hz}$ and for $\rho \in[47 ; 63] \mathrm{kg} / \mathrm{m}^{3}$. Thus we can predict course of damping force for frequencies for which was not measured or for material density of specimens which were not produced. In Fig. 8 there is a simulation of damping force for example for $f=2 \mathrm{~Hz}$ and $\rho \in\{47,49,51,53,55,57,59,61,63\} \mathrm{kg} / \mathrm{m}^{3}$. 


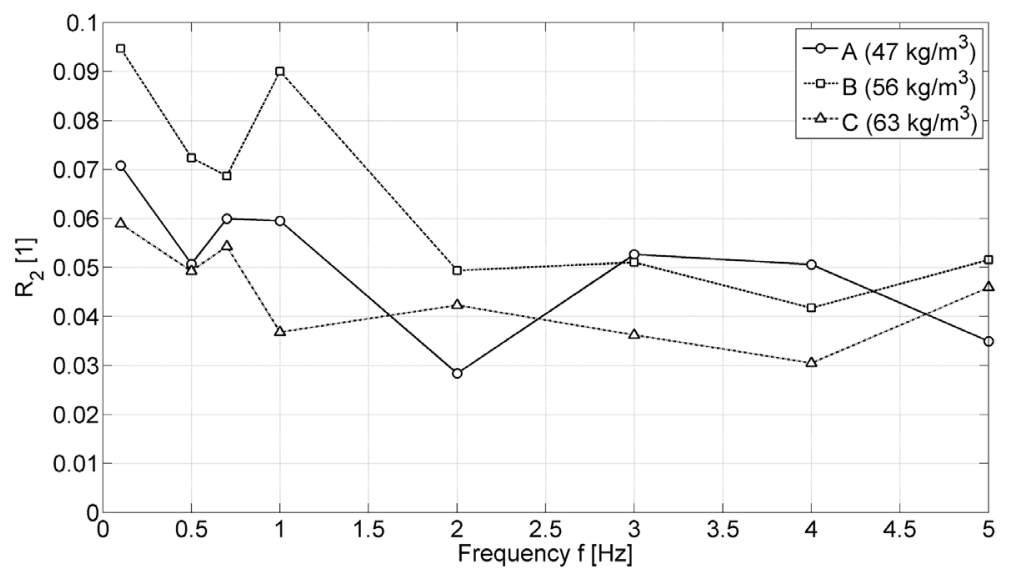

Fig. 7. Simulation error.

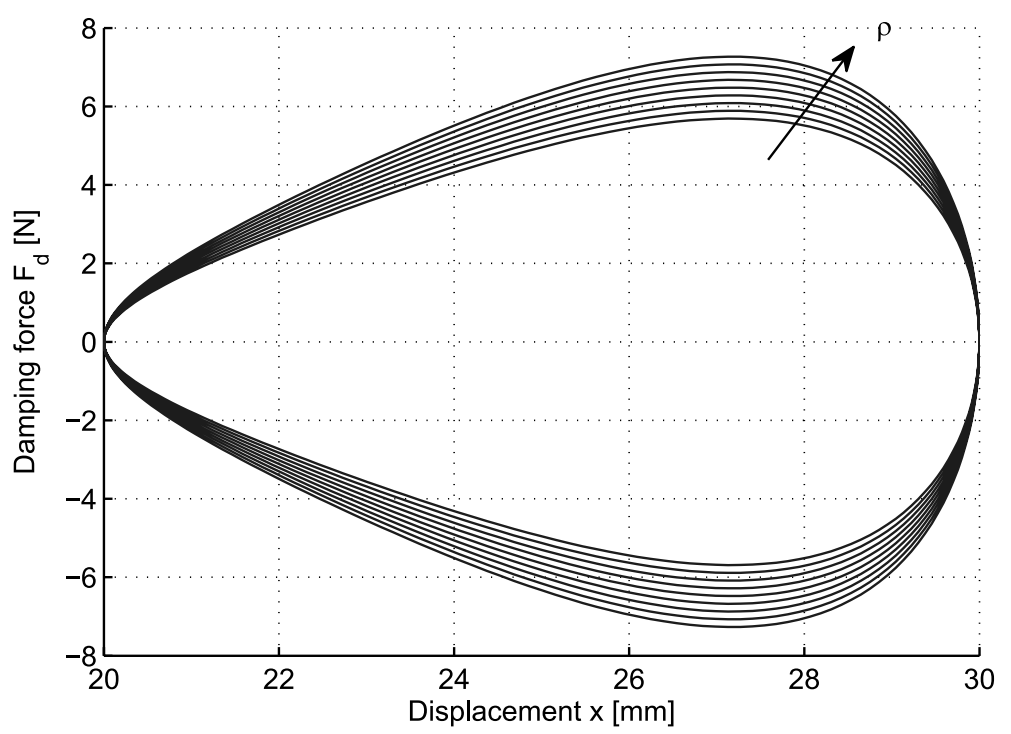

Fig. 8. Prediction of course of damping force.

\section{Conclusion}

In the article the method of simulation of damping force of PU foam specimen was successfully applied. Error of simulation is quantified and is acceptable low. Furthermore, the prediction of damping force was presented. It is possible to use it when we are looking for its course without necessity of measuring it. This prediction works in intervals of frequency of exciting signal $f \in[0.1 ; 5] \mathrm{Hz}$ and material density $\rho \in[47 ; 63] \mathrm{kg} / \mathrm{m}^{3}$.

\section{Acknowledgements}

This work was supported by the subvention from Ministry of Industry and Trade of Czech Republic under Contract Code MPO FT-TA5/102.

\section{References}

[1] D. Cirkl, Mechanical Properties of Polyurethane Foam, Ph.D. Thesis (In Czech), Technical University of Liberec, Liberec 2005. 
[2] D. Cirkl, Measurement and Comparison of Mechanical Properties of Polyurethane Foam Specimens Taken from Different Parts of the Same Car Driver'S Seat, Proceedings of X. International conference on the theory of machines and mechanisms, Technical University of Liberec, Liberec 2008, pp. 141-146.

[3] R. Singh, P. Davies and A.K. Bajaj, Identification of nonliear and viscoelastic properties of flexible polyurethane foam, Nonlinear Dynamics 34 (2003), 319-346,

[4] R. Deng, P. Davies and A.K. Bajaj, Flexible polyurethane foam modelling and identification of viscoelastic parameters for automotive seating applications, Journal of Sound and Vibration 262 (2003), 391-417.

[5] K. Julis and R. Brepta, Mechanics: Dynamics, (Second Edition), Technical Handbook (In Czech), Prague 1984.

[6] R. Brepta, L. Pust and F. Turek, Mechanical Vibrations, technical handbook (In Czech), Prague 1998.

[7] D. Cirkl, Application of Explicit Formulation of Damping Force of Polyurethane Foam in Case of Harmonic Kinematic Excitation, Proceeding of National Conference with International Participation, Svratka 2008. 

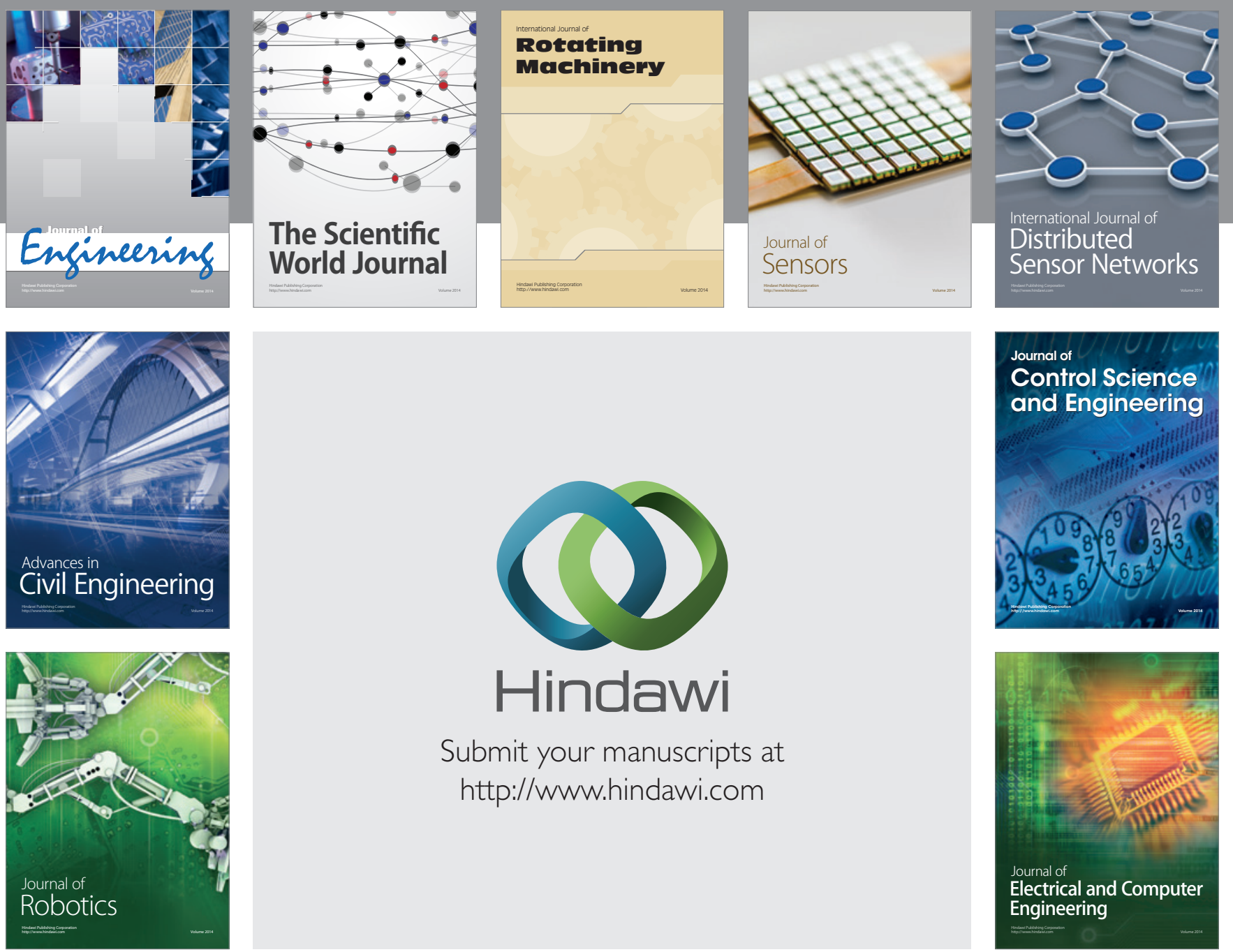

Submit your manuscripts at

http://www.hindawi.com
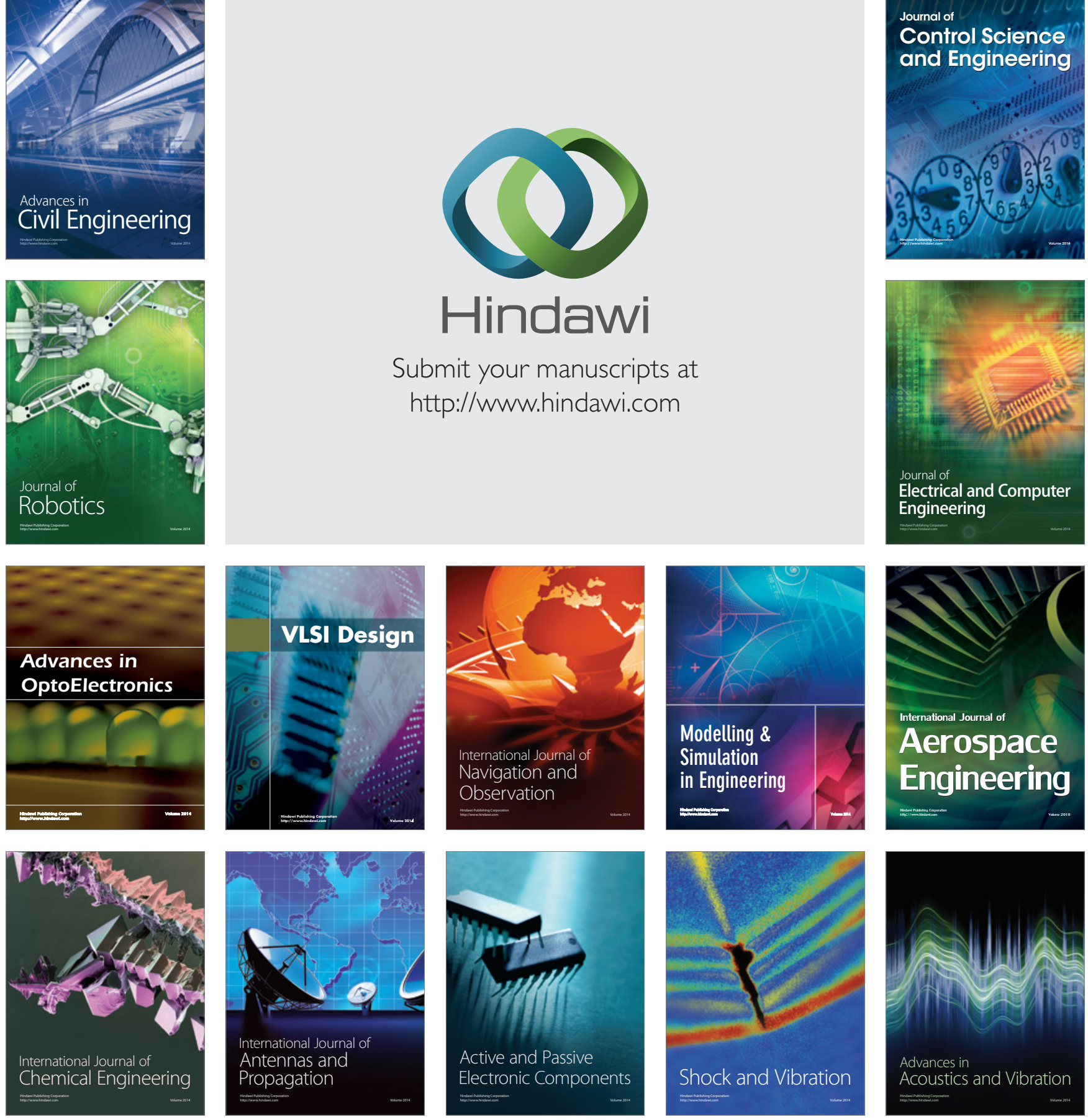